\title{
A Novel S-box of AES Algorithm Using Variable Mapping Technique
}

\author{
Faiz Yousif Mohammad ${ }^{*}$, Alaa Eldin Rohiem ${ }^{* *}$, Ashraf Diaa Elbayoumy ${ }^{* *}$
}

\begin{abstract}
The implementation of a wide, complex and varied networks, let the security development has great importance to the communication innovation. The advance encryption standard (AES) algorithm considered to be the leader of this innovation. A large variety of approaches for modifying AES have been appeared so as to satisfy the varying criteria of different applications [1]. Effort spent on evaluating AES security strength has been very limited, it is significant that the majority of researches at AES dealt with performance evaluation rather than with security evaluation [2].
\end{abstract}

The design and implementation of S-box are representing a key step in the AES algorithm [3]. So many efforts were emulated to redesign, reconstruct or renew the implementation of the S-boxes.

In this paper, AES with Variable Mapping S-box (VMS-AES) is introduced. VMS-AES is a novel AES-like algorithm which uses the key data to generate parameter that used to shift (remapping) the substitution of S-box to another location randomly depend on the initial key and the derived sub keys data. The required modifications are being made for the inverse S-box mapping saving the non-linearity relationship between the S-box and its inverse. VMS-AES allows the change of the system parameters values (number of rounds, key size, chaining mode) then it presents the effects of these changes upon the security and Quality of service (QoS) which is the main objective of the recent work that evaluated the voice over IP (VoIP) security algorithms [4].

Keywords: AES, Cryptography, StsGui, Avalanche effect, Key dependent S-box

\section{Introduction}

In October 2000, after four years effort to replace the aging DES, NIST announced the selection of Rijndael as the proposed AES (NIST 2004). AES is not consummate algorithm but NIST agree on "minor" tweaks of competition submissions [5]. Standardization of AES was approved after public review and comments, and published a final standard FIPS PUB-197 in December 2001. Rijndael submitted by Joan Daemen and Vincent Rijmen, is a symmetric key, iterated block cipher based on the arithmetic in the Galois Field of $2^{\wedge} 8$ elements $-\mathrm{GF}\left(2^{8}\right)$. The input to the encryption and decryption algorithms is a single 128-bit block and three key length alternatives 128,192 , or 256 bits. This key is then expanded into an array of key schedule words: each word is four bytes and the total key schedule is 44 words for the 128-bit key. The input data will be partitioned into a rectangular array of bytes, called a state, the key is similarly pictured as a rectangular array with 4 rows and $\mathrm{N}_{\mathrm{k}}$ columns, where $\mathrm{N}_{\mathrm{k}}$ is equal to the key size divided by 32 . Rijndael round function operates on a state $\mathrm{N}_{\mathrm{r}}$ times, where $\mathrm{N}_{\mathrm{r}}$ is equal to the number of rounds that can be 10,12 or 14 rounds, depending on $\mathrm{N}_{\mathrm{b}}$ and $\mathrm{N}_{\mathrm{k}}$, where $\mathrm{N}_{\mathrm{b}}$ is equal to the block size divided by 32 . Rijndael round is composed of 4 transformations:

1. ByteSub: S-box substitution provides nonlinearity and confusion.

2. Shiftrow: rotations, provides inter-column diffusion.

3. MixColumn: linear combination provides inter-Byte diffusion.

4. AddRoundKey: round key bytes XOR into each byte provide confusion

\footnotetext{
* Sudan Armed Forces, Email: faiz2yousif@yahoo.com, T:0020161076400

** Egyptian Armed Forces
} 
Transformations operations are byte-oriented. Decryption is applying the operations in a reverse order with respect to the order of encryption. For more details refer to [3][6]. Many people have tried to modify AES algorithm to improve its performance, to satisfy the varying criteria of different applications. Some approaches seek to maximize throughput, others minimize power consumption, and yet others minimize circuitry [1]. S-box Construction was attempted by many designers using a fixed irreducible polynomial for higher efficiency and a smaller footprint of the AES intellectual property (IP). For long-term use, however, the fixed irreducible polynomial has been proven to make the system's golden key obvious, thus increasing the decryption rate of confidential files. The decryption methods include side channel, time channel, and power side channel attacks. Some systems can even be decrypted by an inside job [3].

\section{Background}

Techniques for the construction of S-boxes have included pseudo-random generation, finite field inversion and power mappings, and must follow set of criteria, as given by [9-11,14]:

1. Maximization of the nonlinearity so as to provide resistance against linear attacks.

2. No fixed points $(\mathrm{S}-\mathrm{BOX}[\mathrm{x}]=\mathrm{x})$ or reverse fixed points $(\mathrm{S}-\mathrm{BOX}[\mathrm{x}]=\mathrm{x})$.

3. Complexity of the equivalent algebraic S-box description in GF2.

4. Increase the over all security(increase confusion not lead to decrease diffusion).

5. Satisfy the Strict Avalanche Criterion (SAC): If an S-box does not satisfy the SAC, some output bits will be dependent only on some input bits. It is possible to use this dependency in a selected text attack if enough cipher text and plaintext can be obtained. This is useful in key space searches.

6. Output bits change independently i.e. satisfy the Bit Independence Criterion.

7. Many attempts were satisfied some of the above criteria and the more relevant works techniques were discussed below:

\subsection{Key Dependant AES}

Key Dependant AES (AES-KDS) is a technique attempts to make AES key dependent [7]. AESKDS is block cipher in which the block length and the key length are specified according to AES specification: Encryption and decryption process, number of rounds, data and key size were chosen as AES specifications. But the S-box construction was attempt in the following fashion:

1- Initialize the S-box: The first column contains $0 \mathrm{x} 00,0 \mathrm{x} 01, \ldots \ldots, 0 \mathrm{x} 0 \mathrm{~F}$. The second column contains $0 \times 10,0 \times 11, \ldots$ etc, and so on. Thus, the value of the Byte at column $\mathrm{x}$ and row $\mathrm{y}$ is [xy].

2- For each byte value of the key, $\mathrm{k}_{\mathrm{i}}$ (for $0=<\mathrm{I}<=$ key length), for example, if the key length is 16 Byte, the first byte is $k_{1}$, then $k_{2}$ and so on. Examine the value of $k_{i}$, if ( $\left.k_{i} \bmod 2\right)$ equals zero, run a pseudorandom generator for the value of $\mathrm{k}_{\mathrm{i}}$, otherwise run another, also for the value of $\mathrm{k}_{\mathrm{i}}$. Two linear congruence pseudorandom generators are used, called rand 1 and rand 2 that make use of the linear congruence parameters (Michael 2001) for ISO-C Standard and GNU-C respectively.

3- The last run value of the selected pseudorandom generator, $r$, is added to the mean of the key, to introduce a loop counter value for the swapping loop loopcounter $=$ [mean (key) $+r+1]$.

4- Again, use rand 1 and rand2 to generate two byte values that serve as indexes into S-box to select two bytes to be swapped together. This operation continues until the loop counter ends.

5- Repeat steps 2,3 , and 5 until all the key byte values $\mathrm{k}_{\mathrm{i}}$ (for $0=<\mathrm{i}<=$ key length) has been taken.

The inverse substitute byte transformation, called InvSubBytes, makes use of the inverse S-box. The inverse S-box is constructed by determining a substitution pair and replacing it with its inverse [7].

As shown from above discussions, the AES S-box is completely replaced by a new S-box. This eliminates completely Inverse S-box, which violates AES design and hence requires thorough analysis regarding its security, because AES S-box must be tested thoroughly for linear, differential and algebraic attacks. 


\subsection{Pseudorandom S-box Generation Using Chaotic Algorithm}

Two CCS-PRBG were used in design of key dependant S-box, the first one (CCS-PRBG1) was used to generate the 128-bits key. The key is divided into four 32-bits, two of them were used to calculate initial conditions $\mathrm{x}_{1}(0), \mathrm{x}_{2}(0)$ and the remaining two were used to calculate control parameters p1, p2 of CCS-PRBG2. CCS-PRBG2 was used as the source of chaotic keydependant S-box .The output from (CCS-PRBG2) is divided into two words and each word had a length of 8-bit. Each word is decoded to an integer between 0:255. The first output word from the (CCS-PRBG2) is submitted to the S-box. Each output word from the (CCS-PRBG2) is compared with the contents of the chaotic key-dependent S-box in a sequence row by row until the construction of the $16 \times 16 \mathrm{~S}$-box is completed. Repeated words generated from the second (CCS-PRBG2) are discarded [8].

As shown from above, the S-box design strength depends on the random key generation which adds a new time overhead that was needed for the design construction and evaluation.

In general all competing ideas chosen to create good S-box design have one or another outward appearance from the following shortcomings:

- Reconstruct the S-box every execution time decrease the system efficiency and complicates the hardware implementations $[2,3]$.

- Repetitive security evaluation: When a new S-box generated then it need to be tested upon different types of attacks.

- The range of indexes mapping is very limited i.e. every index has only one picture from the substitution domain values. Construction of more than one S-box during one session leads to high overhead.

The above Related works shortcomings become a motivation for new design paradigm in which the above construction overheads must be eliminated or minimized, and mapping domain range must be increased for each S-box element.

\section{A Novel S-box of AES Algorithm Using Variable Mapping}

AES with Variable Mapping S-box (VMS-AES) is block cipher algorithm in which the block length, the key length and the round functions are specified according to AES specification. Figure (2) shows the overall structure of VMS-AES.

A new function was added for Rijndael round transformation which represents a new substitution method in which the substitution is not depend only on input data, but it depend also on the input and derived subkeys. The inverse of the new function was designed to be used in decryption round transformations.

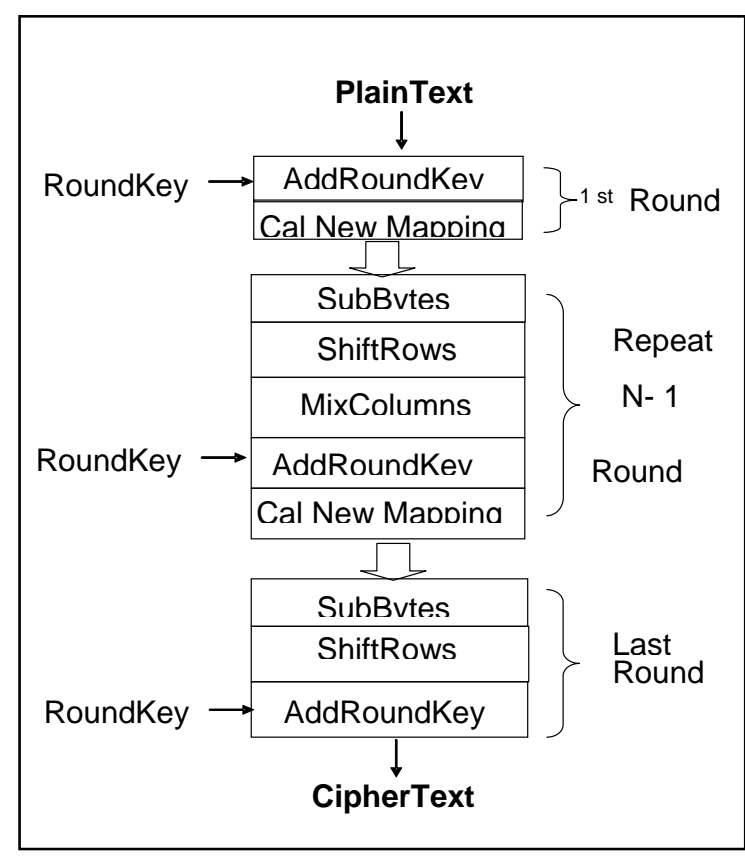

Figure (2a): VMS-AES encryption

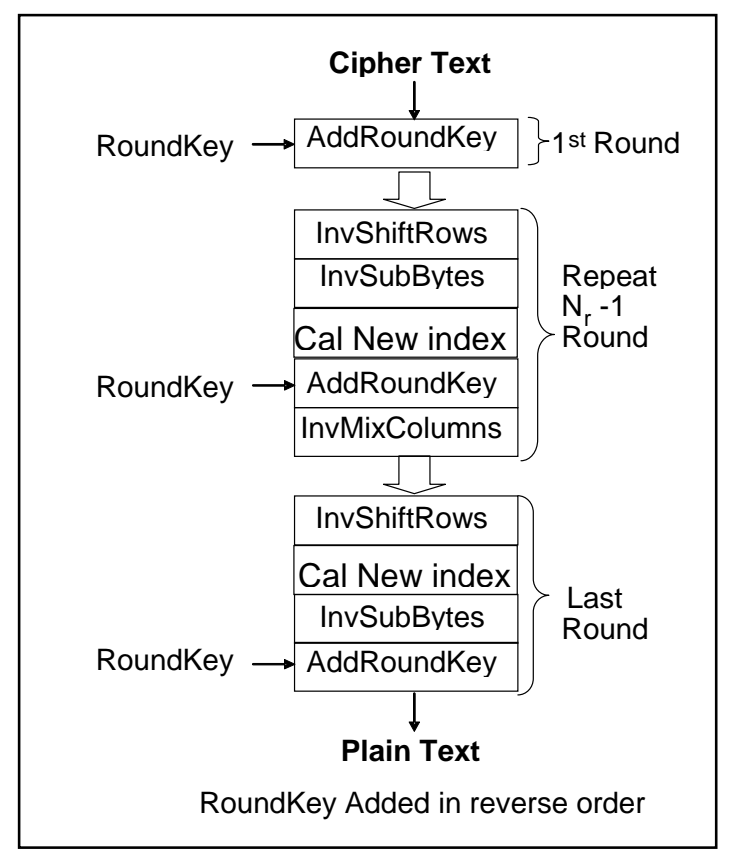

Figure (2b): VMS-AES decryption 


\subsection{New S-box Construction}

Four irreducible polynomials shown in Table (1) were used to create new substitution tables (Sboxes) then they were evaluated so as the best one of them was built-in (VMS-AES). Table (2) and Table (3) describe the S-box and its inverse for the polynomial $\left(X^{8}+X^{6}+X^{5}+X+1\right)$, the other S-boxes and their inverses were appeared in appendix A.

Table (1) different polynomial used to create new S-boxes

\begin{tabular}{|r|rrr|}
\hline \multicolumn{1}{|c|}{ No } & \multicolumn{3}{|c|}{ polynomial } \\
\hline 1. & $\mathrm{X}^{8}+$ & $\mathrm{X}^{6}+\mathrm{X}^{5}$ & $\mathrm{X}+1$ \\
\hline 2. & $\mathrm{X}^{8}+$ & $\mathrm{X}^{5}+\mathrm{X}^{4}+\mathrm{X}^{3}+\mathrm{X}^{2}+\mathrm{X}+1$ \\
\hline 3. & $\mathrm{X}^{8}+$ & $\mathrm{X}^{6}+\mathrm{X}^{5}+$ & $\mathrm{X}^{2}+\mathrm{X}+1$ \\
\hline 4. & $\mathrm{X}^{8}+$ & $\mathrm{X}^{6}+\mathrm{X}^{5}+$ & $\mathrm{X}+1$ \\
\hline
\end{tabular}

Table (2) S-box generated from the polynomial $\left(X^{8}+X^{6}+X^{5}+X+1\right)$

\begin{tabular}{|l|l|l|l|l|l|l|l|l|l|l|l|l|l|l|l|l|l|}
\hline X \\
\hline
\end{tabular}

Table (3) Inverse S-box generated from the polynomial $\left(X^{8}+X^{6}+X^{5}+X+1\right)$

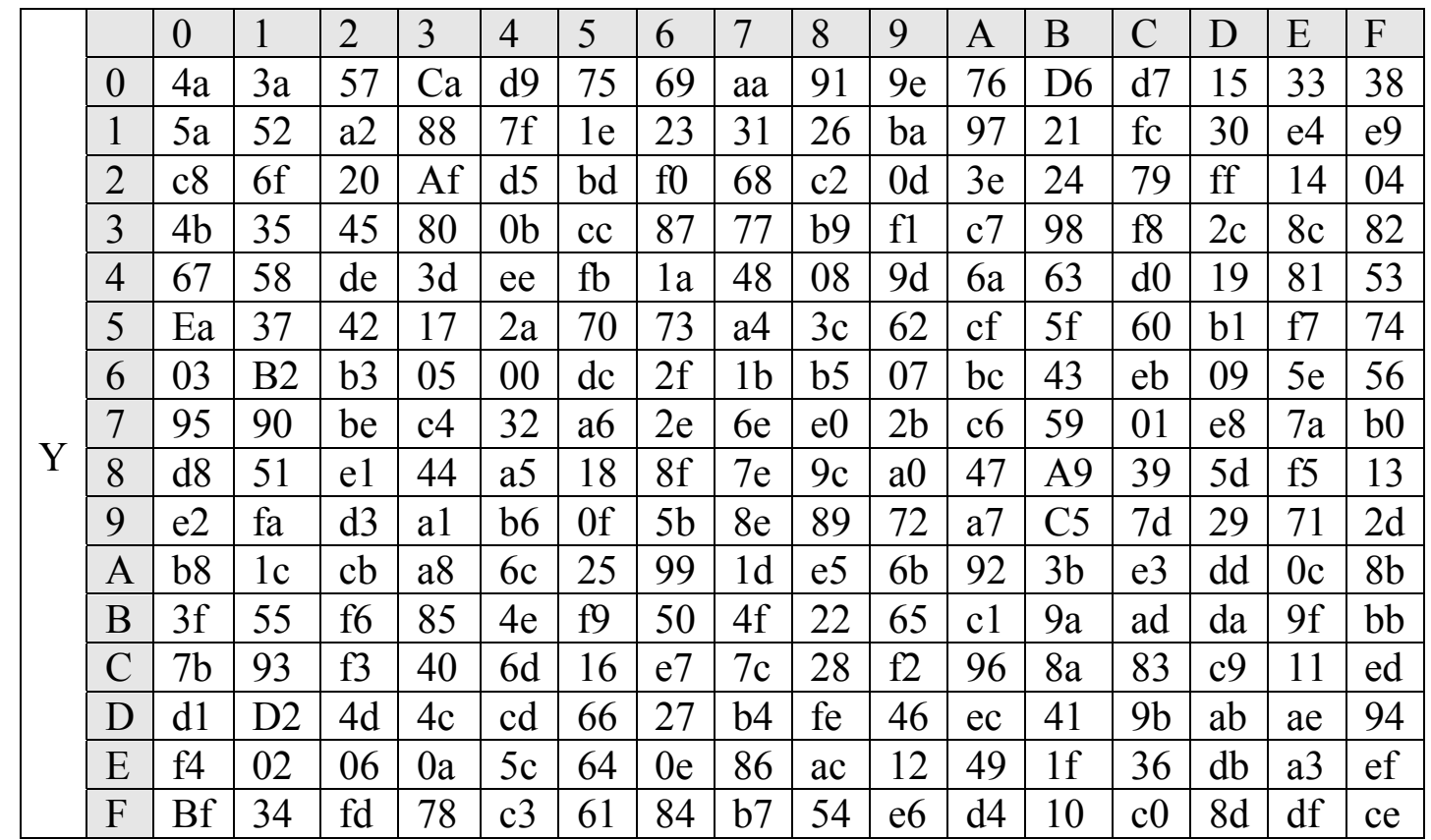




\subsection{AES vs. VMS-AES}

Table (4) represents the main difference between VMS-AES and AES

Table (4) VMS-AES vs AES

\begin{tabular}{|l|l|l|}
\hline The item & AES & VMS-AES \\
\hline Data Block length & 128 Bit & Same \\
\hline Key length & $128 / 192 / 256$ Bit & Same \\
\hline Number of rounds & $10 / 12 / 14$ round & Same \\
\hline Round functions & $\begin{array}{l}\text { ByteSub, ShiftRow, MixColoum, } \\
\text { AddroundKey }\end{array}$ & $\begin{array}{l}\text { Same + CalNewMapping, } \\
\text { CalNewIndex }\end{array}$ \\
\hline Last round & Has no MixColoum & Same \\
\hline S-box & $\begin{array}{l}\text { Fixed ( one to one mapping } \\
\text { function) }\end{array}$ & $\begin{array}{l}\text { New polynomial + New } \\
\text { mapping method }\end{array}$ \\
\hline Mapping to S-box & Depend on the State Byte & $\begin{array}{l}\text { Depend on the State Byte } \\
\text { and on the Keys }\end{array}$ \\
\hline
\end{tabular}

\subsection{VMS-AES S-box mapping Calculation}

S-box substitution represents the main different between VMS-AES proposal and the standard AES. In VMS-AES the forward substitute byte transformation, operate as AES SubBytes function except a shift parameter value would be calculated and added to the index parameter, so as to shift the substitution to new secret location, new because it shift from the specific static location (as in AES) to another location varied from Byte to Byte and this variation does not depend only on the left most and right most of the Byte but it depend also on the SHIFT parameter. The location is secret because it depends on a secret key, and here the substitution stage provides another degree of security in addition of the nonlinearity and confusion which provides from AES style.

Since the property of new mapping is determine the SHIFT value as a one out of 256 (the total number of S-box elements) then it can be consider that as the same as we have 256 S-boxes created from the permutation of the old S-box. The above permutations are not real permutations i.e. all elements are fixed in their positions but only the mapping would be changed in every situation.

Figure (3) represents the substitution and the inverse substitution processes in VMS-AES. Figure (4) shows the mapping range for one byte from the plain text state.

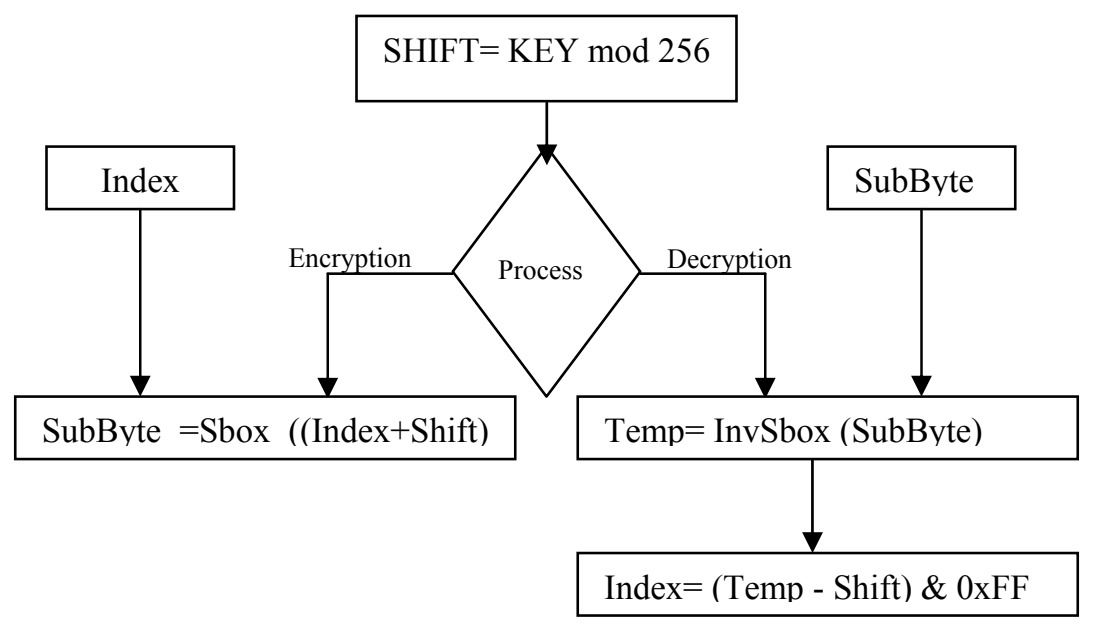

Figure (3): The Substitution and the inverse Substitution in VMS-AES 


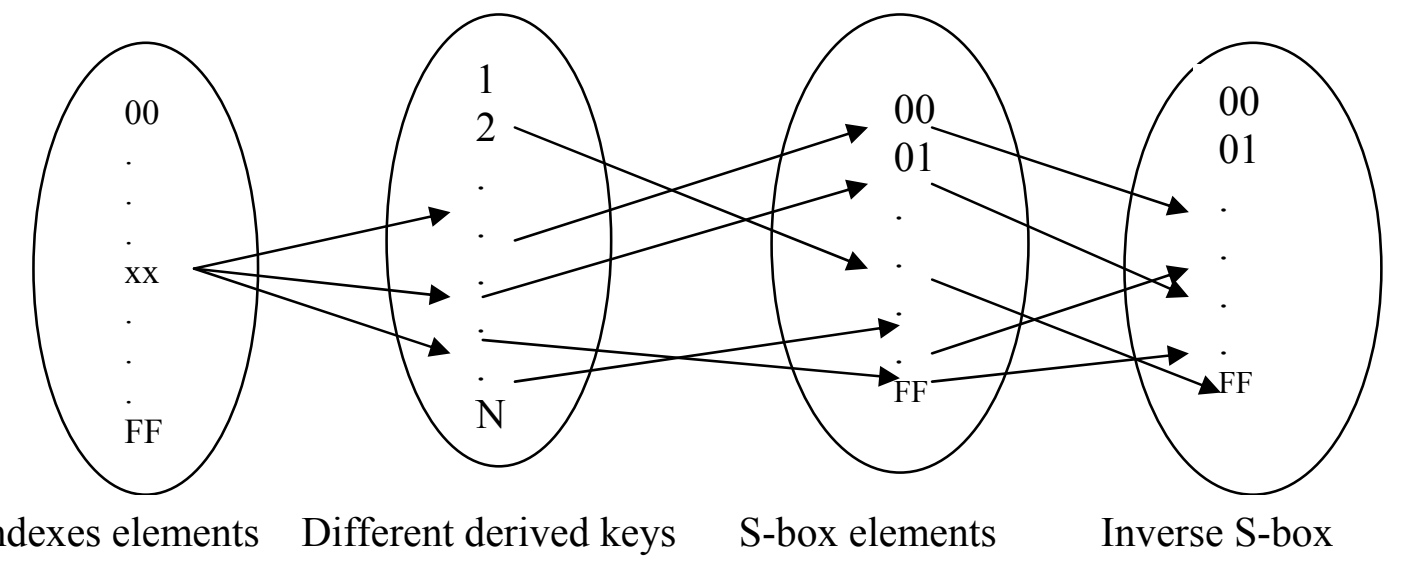

Figure (4): The Mapping range for one byte (index) in

\section{VMS-AES S-box Evaluation}

To evaluate VMS-AES the following verification methods were applied:

1- NIST statistical suite tests.

2- Correlation Coefficient analysis.

3- Avalanche effect measurements.

4- Test strict avalanche criterion.

5- Measurement of Encryption Quality

\subsection{NIST Statistical suite}

The National Institute of Standards and Technology (NIST) develop a Test Suite as a statistical package consisting of 16 tests that were developed to test the randomness of (arbitrarily long) binary sequences produced by either hardware or software based Cryptographic random or pseudorandom number generators. These tests focus on a variety of different types of non randomness that could exist in a sequence. Some tests are decomposable into a variety of subtests [12].

A number of files (with different types) were encrypted using (VMS-AES, AES) algorithms. The encrypted files were entered as inputs parameters to the 16 tests of NIST statistical suite. The average values of the statistical tests for both algorithms were given in Table (5). The tests were applied to a different number of rounds for VMS-AES, and this feature clarifies the VMS-AES flexibility. Note that both proposals (AES \&VMS-AES) were pass all NIST Statistical tests.

Table (5): VMS-AES vs AES Statistical tests average $p$ - values

\begin{tabular}{|c|c|c|c|c|c|c|}
\hline Algorithm $\rightarrow$ & AES & $\begin{array}{l}\text { VMS- } \\
\text { AES }\end{array}$ & $\begin{array}{l}\text { VMS- } \\
\text { AES }\end{array}$ & $\begin{array}{l}\text { VMS- } \\
\text { AES }\end{array}$ & $\begin{array}{l}\text { VMS- } \\
\text { AES }\end{array}$ & $\begin{array}{l}\text { VMS- } \\
\text { AES }\end{array}$ \\
\hline No of rounds $\rightarrow$ & 10 & 10 & 9 & 8 & 7 & 6 \\
\hline \multicolumn{7}{|l|}{ Test Name } \\
\hline Frequency & 0.324 & 0.931 & 0.469 & 0.834 & 0.964 & 0.466 \\
\hline Frequency Test within a Block & 0.195 & 0.275 & 0.568 & 0.074 & 0.074 & 0.637 \\
\hline The Cumulative Sums & 0.811 & 0.740 & 0.407 & 0.437 & 0.991 & 0.740 \\
\hline The Runs Test & 0.178 & 0.804 & 0.834 & 0.932 & 0.804 & 0.195 \\
\hline Longest-Run-of-Ones in a Block & 0.834 & 0.213 & 0.568 & 0.602 & 0.602 & 0.706 \\
\hline The Binary Matrix Rank Test & 0.028 & 0.407 & 0.637 & 0.254 & 0.534 & 0.740 \\
\hline Discrete Fourier Transform & 0.178 & 0.991 & 0.082 & 0.706 & 0.501 & 0.378 \\
\hline Non-overlapping & 0.985 & 0.991 & 0.991 & 0.991 & 0.976 & 0.950 \\
\hline Overlapping Template Matching & 0.407 & 0.350 & 0.772 & 0.134 & 0.637 & 0.044 \\
\hline Maurer's "Universal Statistical" & 0.834 & 0.637 & 0.672 & 0.008 & 0.976 & 0.706 \\
\hline Apen test & 0.178 & 0.602 & 0.178 & 0.378 & 0.706 & 0.911 \\
\hline Random Excursions & 0.706 & 0.985 & 0.973 & 0.862 & 0.862 & 0.941 \\
\hline The Approximate Entropy The & 0.888 & 0.706 & 0.953 & 0.911 & 0.804 & 0.941 \\
\hline The Serial & 0.706 & 0.862 & 0.232 & 0.602 & 0.607 & 0.911 \\
\hline The Linear Complexity & 0.378 & 0.437 & 0.568 & 0.407 & 0.350 & 0.378 \\
\hline Compression ratio & $0 \%$ & $0 \%$ & $0 \%$ & $0 \%$ & $0 \%$ & $0 \%$ \\
\hline
\end{tabular}




\subsection{Correlation Coefficient}

Correlation coefficient is a number between -1 and 1 which measures the degree to which two variables are linearly related. The correlation is 1 in the case of an increasing linear relationship, -1 in the case of a decreasing linear relationship, and some value in between in all other cases, indicating the degree of linear dependence between the variables. If the variables are independent then the correlation is 0 .

Figures below show the correlation distribution of two horizontally adjacent codes in the plaintext/ciphertext for VMS-AES block cipher. Matlab package is used to calculate the Correlation Coefficient in a numerical form and it had a value of $(-0.07491)$ which represent the departure of the plain and cipher text from independence.

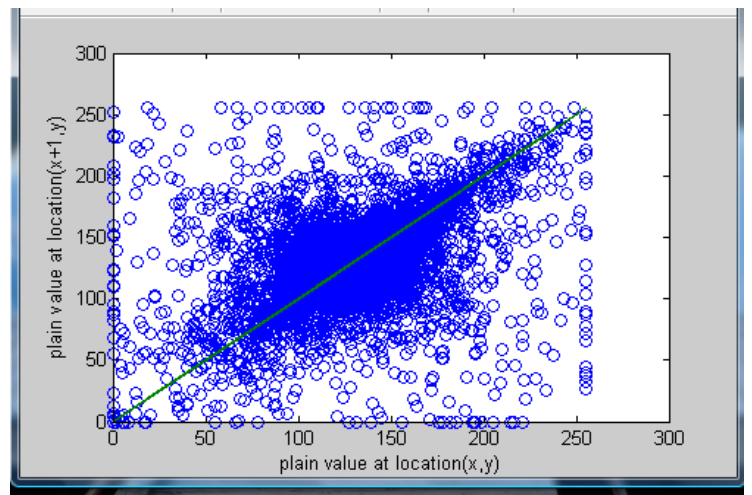

(a)

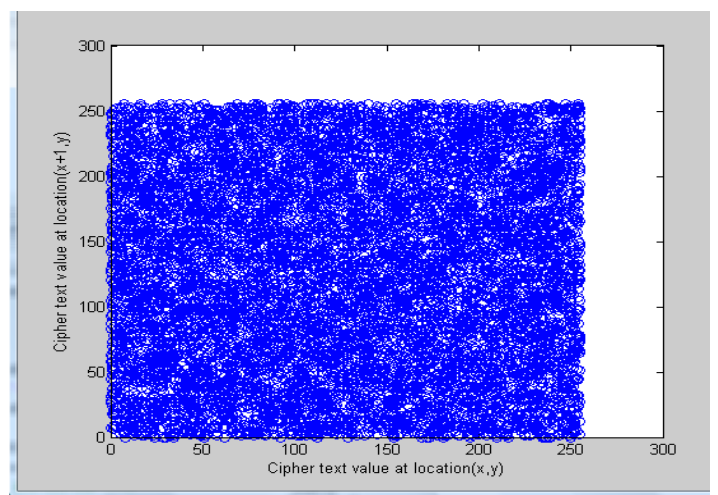

(b)

Figure (5) Correlation Coefficient for selected plain text and cipher text

\subsection{Avalanche effect}

Avalanche effect is a characteristic of an encryption algorithm in which a small change in the plaintext or key give rise to large change in the ciphertext (more than half)[13].

Avalanche effect measurement subsystem was designed and called in recursive mode to calculate the average value of avalanche effect for 20 millions experiments with different random keys used to encrypt a fixed plain text by VMS-AES and AES algorithms simultaneously. Table (6) gives the average results for different number of rounds. The tenth down to the third round pass Avalanche effect test.

Table (6): Avalanche effect percentage for VMS-AES \& AES

\begin{tabular}{|l|l|l|}
\hline No of rounds & AES & VMS-AES \\
\hline 10 & 71.1391 & 74.2860 \\
\hline 9 & 71.1373 & 74.0070 \\
\hline 8 & 69.8731 & 71.9315 \\
\hline 7 & 68.9885 & 71.0221 \\
\hline 6 & 67.5520 & 70.8661 \\
\hline 5 & 65.2132 & 69.9222 \\
\hline 4 & 63.9328 & 67.9723 \\
\hline 3 & 60.1375 & 65.2108 \\
\hline 2 & 16.1436 & 20.2111 \\
\hline 1 & 3.98030 & 4.96690 \\
\hline 0 & 3.92690 & 4.75110 \\
\hline
\end{tabular}

VMS-AES was executed to encrypt a constant plain text and constant key data with all possible S-boxes that could be generated due to the change of shift parameter value (0 .. 256). Different cipher text for every shift value was given. The avalanche effect was calculated and its value was equal or greater than $\mathbf{7 0 \%}$ for all different situations.

\subsection{Strict Avalanche Criterion}

The concepts of completeness and the avalanche effect can be combined to define a new property which we shall call the strict avalanche criterion. If a cryptographic function is to satisfy the strict 
avalanche criterion, then each output bit should change with a probability of one half whenever a single input bit is complemented. A more precise definition of the criterion is as follows. Consider $\mathrm{X}$ and $\mathrm{X}_{\mathrm{i}}$, two n-bit, binary plaintext vectors, such that $\mathrm{X}$ and $\mathrm{Xi}$ differ only in bit $\mathrm{i}, 1$ $\leq \mathrm{i} \leq \mathrm{n}$. Let $\quad \mathrm{V}_{\mathrm{i}}=\mathrm{Y} \quad \mathrm{Y}_{\mathrm{i}}$ where $\mathrm{Y}=\mathrm{f}(\mathrm{X}), \mathrm{Y}_{\mathrm{i}}=\mathrm{f}\left(\mathrm{X}_{\mathrm{i}}\right)$ and $\mathrm{f}$ is the cryptographic transformation, under consideration. The value of bit $i$ in $V_{j}$ (either a 1 or a 0 ) is added to element $a_{i, j}$ in the $m \times n$ dependence matrix A. This procedure is repeated for a large number, $r$, of randomly generated plaintext vectors $X$, and each element in $A$ is divided by $r$. Then each $a_{i, j}$ gives the strength of the relationship between plaintext bit $j$ and ciphertext bit $i$. A value of 1 indicates that whenever bit $j$ is complemented in the plaintext then the ciphertext bit $i$ will also change its value, while a value of 0 indicates that the ciphertext bit is completely independent of the plaintext bit. If all elements in the matrix have a nonzero value then the cryptographic transformation is complete, and if it is to satisfy the strict avalanche criterion, every element must have a value close to one half. Therefore, completeness is a necessary condition if the strict avalanche criterion is to be met.

The strict avalanche criterion matrix given in Table (7) indicates that each element in the strict avalanche matrix has a value close to one-half. So, the new proposal satisfies the strict avalanche criterion.

Table (7): VMS-AES strict Avalanche criterion matrix

\begin{tabular}{|l|l|l|l|l|l|l|l|}
\hline 0.500030 & 0.499822 & 0.499683 & 0.500310 & 0.499879 & 0.499992 & 0.499602 & 0.499757 \\
\hline 0.500028 & 0.499822 & 0.499683 & 0.500310 & 0.499880 & 0.499992 & 0.499601 & 0.499757 \\
\hline 0.500028 & 0.499822 & 0.499683 & 0.500310 & 0.499879 & 0.499992 & 0.499602 & 0.499757 \\
\hline 0.500030 & 0.499822 & 0.499683 & 0.500310 & 0.499880 & 0.499992 & 0.499602 & 0.499758 \\
\hline 0.500030 & 0.499822 & 0.499683 & 0.500311 & 0.499879 & 0.499992 & 0.499602 & 0.499757 \\
\hline 0.500030 & 0.499822 & 0.499683 & 0.500311 & 0.499880 & 0.499992 & 0.499602 & 0.499756 \\
\hline 0.500030 & 0.499822 & 0.499683 & 0.500311 & 0.499879 & 0.499992 & 0.499602 & 0.499758 \\
\hline 0.500030 & 0.499822 & 0.499683 & 0.500311 & 0.499880 & 0.499992 & 0.499602 & 0.499757 \\
\hline
\end{tabular}

\subsection{Measurement of Encryption Quality}

Encryption quality may be expressed as the deviation between the original and encrypted output. Let $\mathrm{F}, \mathrm{F}^{\prime}$ denote the original (plaintext) and the encrypted output (ciphertext) respectively, each of size $M$ samples with $L$ different samples. $F(x, y), F(x, y) \in\{0, . ., L-1\}$ are the different samples of $\mathrm{F}, \mathrm{F}^{\prime}$.We will define $\mathrm{HL}(\mathrm{F})$ as the number of occurrence for each sample $\mathrm{L}$ in the original (plaintext), and HL(F') as the number of occurrence for each sample $\mathrm{L}$ in the (ciphertext). The encryption quality represents the average number of changes to each sample and it can be expressed mathematically as Following

$$
\text { Encryption Quality }=\frac{\sum_{I=0}^{255}\left|H_{L}\left(F^{\prime}\right)-H_{L}(F)\right|}{256}
$$

Table (8) gives results of Encryption quality of AES and its comparison with AES-VMS. From the results we can conclude that the modification to AES will not affect the Encryption Quality of the cipher in any way.

Table (8) Results of Encryption quality of AES and AES-VMS.

\begin{tabular}{|l|l|l|}
\hline No of rounds & AES & VMS-AES \\
\hline 10 & 731.1394 & 734.2881 \\
\hline 9 & 731.1374 & 734.0093 \\
\hline 8 & 698.8735 & 691.9355 \\
\hline 7 & 686.9887 & 701.0521 \\
\hline 6 & 677.5528 & 660.8561 \\
\hline 5 & 659.2139 & 650.9622 \\
\hline 4 & 637.9321 & 670.9783 \\
\hline 3 & 608.1373 & 65.2109 \\
\hline 2 & 169.1435 & 20.2119 \\
\hline 1 & 30.98037 & 4.96690 \\
\hline 0 & 39.92698 & 4.75110 \\
\hline
\end{tabular}




\section{Conclusions}

VMS-AES does not contradict the security, simplicity and easy hardware implementation of AES. The security of AES was improved by employing variable mapping substitution that depends on the secret key which increases the mapping domain. As shown from the results, decreasing the number of VMS-AES rounds to be 7 instead of 10 would not affect the security and would increase the QoS for the applications that are sensitive to QoS like VoIP.

\section{References}

[1] D. Canright,"A Very Compact S-box for AES",Naval Postgraduate School, Monterey CA 93943, USA.

[2] B.preneel,V.rijmen,"Comments by NESSIE project on the AES finalists,NIST,May 2000

[3] Ming-Haw Jing," High Aberrance AES System Using a Reconstructable Function Core Generator",I-Shou University,TAIWAN.

[4] Faiz Yousif, Alaa Eldin Rohiem, A. Elbayoumy, "Security evaluation of VoIP cryptographic algorithms", Proceedings of the 6th ICEENG Conference on Electrical Engineering, 27-29 May, 2008.

[5] Susan Landau, " Communications Security for the Twenty-first Century: The Advanced Encryption Standard",Notice of the AMS 451, APRIL 2000.

[6] F. Granelli and G. Boato," A novel methodology for analysis of the computational complexity of block ciphers: Rijndael, Camellia and Shacal-2 compared", DIT University of Trento,ive 14, I-38050 Trento (Italy).

[7] A. Fahmy, "A proposal For A key-dependent AES", Proceedings of $3^{\text {rd }}$ International Conference: Sciences of Electronic, SETIT 2005, March 27-31, 2005 - TUNISIA.

[8] Rohiem, Elagooz, Dahshan H.,"Anovel approach for designing the S-box of advance ecryption standard using chaotic map", Radio science conference, NRSC May 2005.

[9] G. Boato, "Bijective S-box Applications", Trento (Italy).

[10] Bruce Schneier, "APPLIED CRYPTOGRAPHY.PDF", John Wiley \& Sons ,ISBN: 0471128457, 01/01/96

[11] Alireza,Farmarz," A structure for fast data encryption",Isfahan,Iran,Int. J. Contemp. Math. Sciences, Vol. 2, 2007, no. 29, 1401 - 1424

[12] NIST, "A Statistical Test Suite for Random and Pseudorandom Generators for Cryptographic Applications", NIST Special Publication 800-22, 2003.

[13] William Stallings, "Cryptography and Network Security," Prentice Hall, 2008.

[14] Jennifer Seberry Xian, "Systematic Generation of Cryptographically Robust S-boxes", Wollongong University AUSTRALIA,May 1996. 


\begin{tabular}{|l|l|l|l|l|l|l|l|}
\hline 0.499942 & 0.499943 & 0.499941 & 0.499941 & 0.499943 & 0.499943 & 0.499943 & \\
\hline 0.500739 & 0.500739 & 0.500739 & 0.500737 & 0.500739 & 0.500739 & 0.500739 & 0.500738 \\
\hline 0.500053 & 0.500053 & 0.500054 & 0.500053 & 0.500054 & 0.500053 & 0.500053 & 0.500053 \\
\hline 0.499536 & 0.499536 & 0.499537 & 0.499536 & 0.499537 & 0.499536 & 0.499537 & 0.499536 \\
\hline 0.498904 & 0.498905 & 0.498905 & 0.498905 & 0.498904 & 0.498904 & 0.498904 & 0.498904 \\
\hline 0.499739 & 0.499739 & 0.499739 & 0.499739 & 0.499739 & 0.499739 & 0.499739 & 0.499739 \\
\hline 0.500234 & 0.500234 & 0.500233 & 0.500233 & 0.500233 & 0.500234 & 0.500234 & 0. \\
& & & & & & & 500233 \\
\hline 0.499748 & 0.499748 & 0.499747 & 0.499748 & 0.499748 & 0.499748 & 0.499748 & 0.499748 \\
\hline
\end{tabular}

